\title{
Weltschau und Medizin
}

\section{Max Kälin MD}

Dr. med. Dr. sc. nat., Facharzt für Allgemeinmedizin, Leitender Arzt «Notfall Zürich 3»

\section{Prolog}

Medizin wirkt aus Fassbarem von Wissenschaft und Ingenieurwesen und aus Unfassbarem. Im Kern herrscht Not: Wilhelm Frölich (um 1504 bis 1562) [1], kriegsgetestet: "Angst und Not währt bis in Tod.» Um dieser Mahnung gerecht zu werden, muss Wirken in eine übergreifende Weltschau gebettet sein.

\section{Weltschau und Sehnsucht}

Medizinische Weltschauen: die schamanische, das Corpus Hippocraticum, die wissenschaftliche im Kontrast zu Aberglaube [2], die homöopathische, andere. Sehnsüchte: Gerechtigkeit, Heilung, Schutz, Vollkommenheit, Errettung [3]. Jurist, Ärztin, Krieger, Künstlerin sind Hoffnungsträger der ersten vier. Mit der fünften, der Errettung in ein Dort ohne Übel, ist es schwieriger. Das Heilige kann helfen, aber das ist vielen fremd. Es braucht Mittler wie Buddha oder Jesus oder persönlich erarbeitete Gewissheit der Irrelevanz derartiger Mittler.

In der Medizin herrschen Angst und Not wie je. Eine erarbeitete und durch Erfahrung validierte Weltschau kann helfen, dieser Misere zu begegnen. So können durch Deutung Sinn und Trost entstehen. Deutung, umfassend und tiefgründig, ist mehr als Erklären von Pathologie oder eigenem Zutun. Deuten beinhaltet auch das Einbetten des Schicksalhaften in die Vergänglichkeit des Seins.

\section{Komplexität}

Das Formen einer Weltschau geschieht durch Denken, eine Funktion des menschlichen Hirns, der komplexesten bekannten Struktur des Universums (Masse um 1500g, Anzahl Neuronen und Gliazellen je um $90 \times 10^{9}$, Anzahl Synapsen als Endstellen der Signalübertragung des Konnektoms wesentlich mehr [4]). Komplexität des Hirns ermöglicht das Staunen und Fragen, das Kritisieren führt zum Prüfen und das Misslingen irgendwelcher Falsifikation unterstützt eine zunehmend vertrauenswürdige Sicht der Wirklichkeit. Das Hirn als lebendes Substrat der wissenschaftlichen Methode ist in seiner Komplexität Voraussetzung für das Generieren einer Weltschau, ob genügend ist offen.
Es sieht aus, als hätte im Universum Komplexität, Typ Homo sapiens, entstehen müssen, um was, wann, wo herauszufinden. Das Universum auf dem Weg zur Selbsterkenntnis mit Komplexität als Methode. Das kann extraterrestrisch angefangen haben. Homo sapiens als erster Träger derartiger Fähigkeit wäre erstaunlich. Das würde heissen das Universum als Ganzes auf dem Marsch, ein Prozess von einmal hier, einmal dort, angewiesen auf Goldilocks' Planeten [5], die über ein paar Milliarden Jahre Schutz geboten hätten, um entsprechend fähige Wesen werden zu lassen [6]. Dem Prozess entspräche ein Gefunkel von Erkenntnis durch die Galaxien, ein wenig hier, ein wenig dort, mit der Möglichkeit des Austauschs: das Universum ein Metahirn, Konnektom unbekannt.

\section{Ärztliche Betrachtung: Misere}

Damit kommen wir dem ärztlichen Alltag näher. Niemand mit Erfahrung ist "so verrückt, so nicht folgerichtig, niemand wird mir das abnehmen»; unbekannt. Genauer: Es ist das Alltägliche, und es ist so, weil wir Menschen komplex sind. Hier haben Raum und Zeit den Vorsprung einer hochentwickelten Metrologie. Diese fehlt der Komplexität: Das sich aufdrängende Mass wäre Entropie, oder ähnlich. Sicher ist das menschliche Hirn ein lokales Entropieminimum. Es sieht aus, als würde das Universum bei zunehmender Gesamtentropie - und assoziiert, drohendem Wärmetod - sich genau über dieses Schicksal durch Komplexität klar werden zu wollen. Der Gedanke eines letztlichen Wärmetods, formuliert durch Thomson (1824-1907), geht auf Clausius zurück (1822-1888). Er vollendete die Thermodynamik und prägte den Entropiebegriff [7-9].

Damit kommen wir einem Aspekt des Alltags näher, dem Leiden. Es ist zentrale Verantwortung der Medizin, Leiden zu erkennen und zu lindern, keinen Schaden zu setzen: Im Westen: primum non nocere (lateinisch: «erstens nicht schaden»), im Osten: ahimsa (Sanskrit: «Nicht-Verletzen, Gewaltlosigkeit») [10]. Die Erkenntnis, dass alles eines Tages nicht mehr sein könnte, wirklich alles sich in einer Suppe von Elementarteilchen entdifferenzieren könnte, auch wenn es noch ein Weilchen dauerte, ist enorm kränkend. Schö- 
nes und Wüstes, Grausames und Heiliges - alles für immer nicht mehr. Tröstlich, es wäre auch das letztliche Ende allen Leidens.

Damit zu einem weiteren Aspekt des Alltags, der Prognose. Fehlleistungen sind häufig, schmerzlich und der Komplexität immanent. Die Prognose obigen Endes aller Komplexität in einer Elementarteilchensuppe ist eine wissenschaftliche. Allerdings übertrifft diese Prognoseform alle anderen um Längen. Durch Erkenntnis zur Prognose ist eine aussergewöhnliche Menschheitsleistung, ein Prozess, der sich immer in der Vergangenheit verankern muss, um die Zukunft vorauszusagen. Da die Signalgeschwindigkeit durch die Lichtgeschwindigkeit begrenzt ist, oder so zu sein scheint, ist alles Messen zum Zeitpunkt der Anwendung immer Vergangenheit. Sobald wir uns etwas Gegenwärtigem bewusst werden, ist es Vergangenheit. Messungen abgeschlossen, aufgezeichnet und mit dem Lineal extrapoliert, und schon ist der Datensatz veraltet. «Die Sonne steht am Himmel», ist eine Prognose: "Vor gut 8 Minuten stand sie dort, wo sie jetzt steht», wäre herauszufinden. Erst in gut 8 Minuten wissen wir, ob sie zum Beobachtungszeitpunkt noch existierte.

Damit zu einer wichtigen Prognose, der Prognose des Lebensendes. Üblicherweise wollen wir leben, wir klammern uns an Existenz. Diese hat ein Ende, aber: «Warum bei mir? Warum nicht nur bei den anderen?» Diesem Dilemma ist mit Respekt zu begegnen: Spirituelle Erfahrung wird massgebend.

\section{Ärztliche Betrachtung: Spiritualität}

Spirituelle Erfahrung ist verbreitet, aber zu deuten heikel. Der Begriff ist schwierig und vage: Das Lateinische spiritus (Geist, Hauch) impliziert etwas wie durch das Universum Wehendes, ob ein Etwas oder Nicht-Etwas ist offen. Buddha und Jesus hinterliessen Weisungen, wie ein Mensch durch Praktizieren dem Geheimnisvollen näher kommen kann. Zu dessen Wesen schwiegen beide. Buddha lehrte den "Achtfachen Weg» (Pali: ariya-atthangika-magga) [11] und Jesus vorzugsweise durch Gleichnisse, mit der Mahnung, das Selbst letztlich aufzugeben [12]. Beide rieten, durch Üben sich so zu wandeln, dass Klarheit zunimmt und schädigendes Denken abnimmt. Das durch Denken Schädigende kann an Konsequenzen entsprechender Handlungen erkannt werden. Je weniger selbstsüchtig das Handeln, desto weniger kaputt das Geschirr. Klarheit begünstigt Vollbremsung vor dem Abgrund.

Die Wissenschaft hat enorm beigetragen, Not zu lindern, und ist auch ein Werkzeug, um ihr zu begegnen. Aber der Not einen Sinn zu geben scheint durch ihren Anspruch auf Objektivität unmöglich zu sein. Zwei
Beiträge aus der Antike helfen weiter: Prediger 9:11 aus dem Alten Testament (AT) und das Kalama Sutta. Prediger 9:11 in Luther's Übersetzung [13].

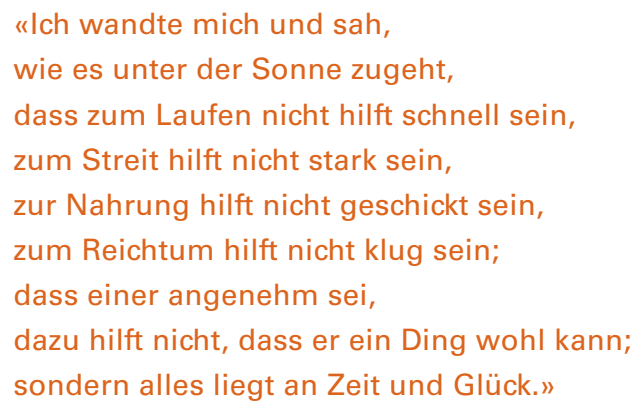

Beobachter des Lebens werden zustimmen: Genau so verkehrt ist es. Allerdings ist es auffälliger, wenn stark durch schwach ausgehebelt wird oder gut durch böse, als wenn stark oder gut obsiegt. Glück und Unglück können jedem zustossen. Gut geht nicht mit gut, und böse nicht mit böse. Wenn Ärztinnen und Ärzte diesem Umstand begegnen, sind sie gefordert, ihn in eine erträgliche Form zu wandeln, oft durch Sinnsuche. Häufig misslingt das, und dann bleibt nur Erdauern. Diese therapeutische Anstrengung setzt Verankerung in einer erarbeiteten und gelebten Weltschau voraus. Buchstabengelerntes und Schwafeln versagen.

«Glück» bedeutet hier eher Zufall als Glück versus Unglück. Zufall ist, was zufällt, und das AT lässt keinen Zweifel bezüglich des Agens, das zufallen lässt, was uns zusteht, es ist Yahweh, Gott der Hebräer. Das AT hat in Hiob [14] einen Vorschlag, wie Übel zu begegnen sei. Hiob, gerecht und fromm, hatte alles, Häuser, Ländereien, Frauen, Kinder, Gesundheit. Er verlor alles, als Folge einer Wette zwischen Yahweh und Satan, und gewann alles durch Treue zu Gott und unerschütterlichen Glauben zurück.

Derartige Standhaftigkeit ist rar: Es gibt Menschen, die unerschütterlich blind glauben oder blind Lehren vertrauen können, die ein Leben führen, ohne Glas zu zerbrechen, und friedlich sterben. Das Übliche jedoch ist Interesselosigkeit an existentiellen Aspekten und Unwissen. Après moi le déluge. Was nach mir kommt, hat nichts mit mir zu tun. Verantwortung entstanden durch mein Handeln - vergiss es. Sterben ein Graus. Wenn guten Menschen Übles geschieht und vice versa, erklärt das die hindu-buddhistische Tradition mit Karma, einem Modell, das Handlungen auf allen Ebenen mit Konsequenzen verknüpft, und über viele Existenzen. Der innere Mechanismus, dieses Karussell von Kommen und Gehen, ist paticcasamuppada (Pali) [15], Buddhas Schlüsselerfahrung. In diesem Kontext ist das Kalama Sutta bedeutsam [16]. Es enthält neun Warnungen: 
Kalamas, lässt euch nicht täuschen durch

Hörensagen

Überlieferungen

Meinungen

Autorität der Schriften

logische Folgerungen

Folgerichtigkeiten basierend auf Mutmassungen

Nachdenken über Scheinbares

Möglichkeiten

Autorität eines Meisters

In den Schriften der Menschheit ist derartiger Rat einzigartig: Mensch, deine Haltung sei skeptisch, da deine spirituelle Basis nicht anders sein kann als deine eigene persönliche Erfahrung. Letztlich begleitet dich einzig diese durch Leben und Sterben.

Die skeptische Haltung im Umgang mit dem inneren Weg ist dieselbe wie der Umgang mit dem äusseren Weg, der Wissenschaft. Beim Ersten reflektiert das beobachtende Ich das innere Universum, beim Zweiten das äussere. Beim Ersten ist der Mensch allein mit den Anleitungen der Menschheit, wie das zu bewerkstelligen wäre. Beim Zweiten muss er sich austauschen, da wissenschaftliche Erkenntnis nur durch externe Prüfung gültig werden kann. Bei beiden Anstrengungen sind der Fallstricke wegen Wegbegleiter wichtig.

Zum inneren Weg hat die Menschheit einiges entdeckt: Pilgerfahrt, Gebet, Klausur, Meditation, Rückzug, monastische Lebensweise und andere [17]. Derartiges Praktizieren begünstigt die Sicht nach Innen, und die ist von Nöten, wenn die Felle gesicherten ärztlichen Handelns davonschwimmen.

Vorschlag: Auslegeordnung der Methoden des inneren Weges, sich für Gangbares entscheiden, dieses praktizieren. Nichts Spektakuläres ist zu erwarten, aber die inneren Veränderungen werden bei der Begegnung mit dem Hässlichen, dem Bösen, dem Furchtbaren bemerkbar.

\section{Epilog}

Heraklit wird «alles fliesst» ( $\pi \alpha v \tau \alpha \rho \varepsilon l)$ zugeschrieben. Diese Ansicht wurde von Whitehead (Mathematiker und Philosoph; 1861-1947) [18] exploriert. Zusammengefasst: Alles ist Prozess. Das würde heissen: Wenn alles fliesst, gäbe es nichts, das nicht fliesst, nur das Nichts würde nicht fliessen und hätte, da keinem Wandel unterstellt, keine inhärente Zeit.

Eingebettet in diesen Prozess entwickelt sich im Menschen Weltschau, sekundiert durch Werkzeuge wie wissenschaftliche Methode, Religion, moralische Codices, ein lebenslanger Subprozess.

Im Umgang mit Furchtbarem sind Ärztinnen und Ärzte gefordert durch Wissen, Können und Weltschau, gebün- delt und kongruent. Allen dreien liegt dasselbe zugrunde: üben, üben, üben. Langweilig? Pessimistisch? Dieses Dreibein hält Stand und fördert innere Freiheit: Eigene Angst und Hilflosigkeit, da gemindert, tragen weniger zum Übel bei. Handlung in extremis wird besser durchgestanden.

\section{Literatur und Anmerkungen}

1 https://commons.m.wikimedia.org/wiki/File:Wilhelm_Froelich jpg/26 Dec 2017.

2 Wissenschaft setzt Glauben an die Existenz von Ordnung in der Natur voraus, und an die Möglichkeit, diese zu entdecken. Dieser Glaube ist nicht Aberglaube, nicht blind: Sich eines Besseren belehren lassen ist Kern des Vorgehens.

3 Carl Gustav Jung (1875-1961) prägte dazu die Vorstellung Archetyp

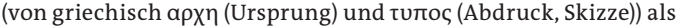
etwas geistig Strukturelles mit einer Tendenz, sich in Vorstellungen zu manifestieren. Struktur und Manifestationstendenz wären unbewusst. Archetypen sind im Vorfeld der Testbarkeit und so keine Hypothesen.

4 Herculano-Houzel S. The human brain in numbers: a linearly scaled-up primate brain. Frontiers in HUMAN NEUROSCIENCE. 2009;3:31. https://www.ncbi.nlm.nih.gov/pmc/articles/ PMC2776484/1 Jan 2018.

5 Planeten innerhalb lebensermöglichender Bedingungen (nach "Goldilocks and the Three Bears»). http://www.crystalinks.com/ goldilocksplanets.html/2 Jan 2018.

6 Die Häufigkeit von Sternen mit Planetensystemen beginnt bekannt zu werden. Per 1. Jan 2018 sind 3726 Exoplaneten in 2792 Systemen bekannt (https://en.wikipedia.org/wiki/

Exoplanet\#Confirmed_discoveries/2 Jan 2018). Es ist zu erwarten, dass Goldilocks-Häufigkeiten in naher Zukunft ebenfalls bekannt werden. Diese könnten dann die Plausibilität extraterrestrischer Intelligenz vom «Erde-Typ» unterstützen. Der letzte Schritt wäre der Kontakt mit derartiger Intelligenz.

7 Clausius R. Über verschiedene für die Anwendung bequeme Formen der Hauptgleichungen der mechanischen Wärmetheone. Naturforschende Gesellschaft Zürich, Vierteljahrsschrift. 1865; 10(1):1-59. http://www.ngzh.ch/publikationen/vjs/10/1/1 Jan 2018.

8 Gassmann F. Rudolf Clausius vollendet die Thermodynamik. Naturforschende Gesellschaft Zürich, Vierteljahrsschrift. 2016; 161(2):17-8. www.ngzh.ch/media/vjs/161_2_17-18.pdf/1 Jan 2018.

9 Rudolf Clausius fasste seine Ausführungen zur Thermodynamik 1865 zusammen: «... Grundgesetze des Weltalls ... Die Energie der Welt ist konstant. Die Entropie der Welt strebt einem Maximum zu.»

10 Der Ursprung von primum non nocere ist unklar. Als Prinzip aber im Corpus Hippocraticum enthalten und zur Weltethik gehörig. Ahimsa, im Prinzip dasselbe, hat aber in der hindu-buddhistischen Tradition eine erweiterte Deutung: Durch Verletzen von ahimsa entstehen karmische Konsequenzen, die wieder ausgebadet werden müssen.

11 Das erste Glied ist Samma Ditti (Pali), ungefähr «Die rechte Sicht», und bedeutet das Erkennen davon, was heilsam ist und was nicht. Diese Einsicht ermöglicht die Wahl der Marschrichtung.

12 Beispielsweise elaboriert in «Imitatio Christi» von Thomas von Kempen (um 1380 bis 1471). Quelle (Original mir nicht zugänglich): https://portal.dnb.de/opac.htm?method=showFullRecord\&curren tResultId $=\% 22118642766 \% 22+$ sortBy+jhr\%2Fsort.ascending $\% 26$ any \&currentPosition=0/21 Jan 2018.

13 Deutscher Evangelischer Kirchenausschuss, Version 1951.

14 Jastrow M. The Book of Job: Its Origin, Growth and Interpretation. J. B. Lippincott Company. 1920:369.

15 Analysis of Dependent Co-arising; translation from Pali by Thanissaro Bikkhu. https://www.accesstoinsight.org/tipitaka/sn/sn12/ sn12.002.than.html/4 Feb 2018.

16 (Kesamutti [aka Kālāmā] Sutta (AN 3.66). https://www.buddhavacana.org/sutta/anguttara/03/an03-066.html/3 Feb 2018.

17 Saulus ritt nach Damaskus und hatte eine Jesus-Erfahrung. Die Me thode war nicht etwa Reiten: Die Bedingungen waren reif für sein Erlebnis, dieses geschah, und als es geschah, war er beim Reiten.

18 Whitehead AN (Gifford Lectures: 1927-1928). Process and Reality (An Essay in Cosmology). New York: The Free Press (Paperback Edition 413); 1985 